\title{
Levels of growth factors from platelet-rich fibrin from chronic periodontitis versus periodontally healthy subjects: a pilot study
}

Jennifer Chang DDS, MSD ${ }^{1}$

Steven B. Blanchard DDS, MS²

L. Jack Windsor $\mathrm{PhD}^{3}$

Richard L. Gregory $\mathrm{PhD}^{3}$

Yusuke Hamada DDS, MSD²

\section{Affiliations:}

${ }^{1}$ Department of Periodontics and Dental Hygiene, University of Texas Health Science Center at Houston School of Dentistry, USA

7500 Cambridge Street, Houston, TX 77045

${ }^{2}$ Department of Periodontics and Allied Dental Programs, Indiana University School of Dentistry, Indiana, USA

${ }^{3}$ Department of Biomedical and Applied Science, Indiana University School of Dentistry, Indiana, USA

1121 W Michigan Street, Indianapolis, IN 46202

\section{Correspondence author:}

Dr. Yusuke Hamada; yuhamada@iupui.edu; Phone: 317-274-5121; Fax: 317-274-7557.

\begin{abstract}
Objectives This study aimed to: 1) compare the amounts of growth factors from platelet-rich fibrin (PRF) between chronic periodontitis and periodontally healthy subjects; and 2) evaluate the relationships between the amounts of growth factors from PRF with complete blood counts (white blood cell (WBC) and platelet counts) and the serum concentrations of IL-1 $\beta$, IL-6, and TNF- $\alpha$.

Materials and methods Venous blood was collected from chronic periodontitis (test) and periodontally healthy subjects (control). PRF and serum were collected from the centrifuged blood. Liquid exudates from the compression of PRF were collected. The compressed PRF membranes were incubated in saline and eluted aliquots were collected at 1, 24, and 72 hours, and the membranes were then digested with trypsin. EGF, IGF-1, PDGF-BB, TGF- $\beta 1$, and VEGF in the exudates and eluents were quantified by ELISA. Serum was used for IL-1 $\beta$, IL-6, and TNF- $\alpha$
\end{abstract}

This is the author's manuscript of the article published in final edited form as:

Chang, J., Blanchard, S. B., Windsor, L. J., Gregory, R. L., \& Hamada, Y. (2020). Levels of growth factors from platelet-rich fibrin from chronic periodontitis versus periodontally healthy subjects: A pilot study. Clinical Oral Investigations, 24(2), 823-832. https://doi.org/10.1007/s00784-019-02944-7 
quantification. Complete blood counts were measured.

Results There were no significant differences in the amounts of growth factors from PRF exudates and membranes measured between groups (all $\mathrm{p}>0.05)$. The test group had significantly higher $\mathrm{WBC}(\mathrm{p}<0.05)$. However, there was no significant correlation between the WBC and the amounts of the growth factors from PRF (all $\mathrm{p}>0.05$ ).

Conclusions PRF can be utilized as an autologous source of growth factors not affected by periodontal condition and WBC level.

Clinical relevance The amounts of growth factors from PRF were not affected by the periodontal condition of the patient.

\section{Key Words}

Platelet-Rich Fibrin; Platelet concentrates; Chronic Periodontitis; Growth Factors

\section{Acknowledgments:}

The authors would like to thank the support from a Young Researcher Grant provided by the Osteology Foundation, Switzerland (project number: 16-142), and the Indiana University School of Dentistry Graduate Student Research Committee. We would also like to thank Mr. George Eckert for the statistical analyses. 


\section{Introduction:}

The most recent National Health and Nutrition Examination Survey estimates that $47.2 \%$ of the U.S. population between the ages of 30 to 70 years old are affected by periodontitis [1]. Periodontitis is a microbial biofilm-induced inflammatory disease, causing periodontal tissue destruction and eventual tooth loss. The inflammatory status of periodontitis is associated with systemic elevation of pro-inflammatory cytokines such as interleukin-1 $\beta$ (IL-1 $\beta$ ), IL6, and tumor necrosis factor- $\alpha(\mathrm{TNF}-\alpha)$ [2]. Current treatment regimens utilize a variety of techniques and materials attempting to regenerate soft and hard tissues around the teeth and the resultant alveolar ridge defects from destructive periodontal disease [3, 4].

Platelet-rich fibrin (PRF) is categorized as a second-generation platelet concentrate, which is collected from centrifuged blood of patients without the use of any anticoagulants resulting in initiation of the natural coagulation cascade by platelets with fibrinogen being transformed by thrombin into fibrin. After centrifugation, the fibrin clot is obtained between the erythrocytes at the bottom and the acellular serum at the top due to density differences. Platelets entrapped within the fibrin clot release growth factors upon activation and degranulation [5-9]. Results from previous studies revealed that autologous blood concentrates are a safe and convenient source to deliver polypeptide growth factors such as epidermal growth factor (EGF), insulin-like growth factor-1 (IGF-1), plateletderived growth factor-BB (PDGF-BB), transforming growth factor- $\beta 1$ (TGF- $\beta 1$ ), and vascular endothelial growth factor (VEGF) [10, 11]; all of which can enhance wound healing and periodontal regeneration. The advantages of utilizing PRF as an autologous source of growth factors compared to other allograft or xenograft sources are that concerns of disease transmission and adverse immunogenic reactions are eliminated. Numerous studies have evaluated the benefits of clinical application of PRF for periodontal regenerative therapies and reported improvements in clinical periodontal parameters and the enhanced healing of soft and hard tissues [12-14]. As an autologous material, patient-related factors may affect the quantity and quality of the PRF. For example, previous study demonstrated that older and female patients could produce larger PRF membranes [15]. However, very few studies have been done evaluating patient-related factors affecting the quantity and types of growth factors from PRF. There is no available information to determine if the platelet count or the inflammatory status of patients with chronic periodontitis may influence the levels of growth factors present and released over time from the PRF. Platelets are the source of many of the known growth factors released from PRF [16]. Furthermore, previous studies have demonstrated several possible links of periodontitis to systemic diseases with evidence of systemic 
inflammatory responses which could either activate platelets to release growth factors [17], or possibly with inflammation, healing response may be down regulated, with decreased amounts of growth factors from PRF. Therefore, the objectives of this study were to: 1) compare the amounts of growth factors (EGF, IGF-1, PDGF-BB, TGF- $\beta 1$, and VEGF) from PRF between chronic periodontitis and periodontally healthy subjects; and 2) evaluate the relationship between the amounts of growth factors from PRF with the complete blood count (white blood cell and platelet counts) and the concentrations of IL-1 $\beta$, IL-6, and TNF- $\alpha$.

\section{Materials and Methods:}

\section{Subjects:}

All subjects were recruited from the Graduate Periodontics Clinic and the Comprehensive Care Clinics of Indiana University School of Dentistry with approval from the Indiana University Institutional Review Board (protocol\# 1704165172) between September to November 2017. Subjects were screened to confirm that they met the inclusion criteria and written informed consents were obtained. The study group were patients with a diagnosis of generalized moderate to severe chronic periodontitis. Based on the 2015 American Academy of Periodontology Task Force report on the update to the 1999 classification [18], patients with greater than $30 \%$ of teeth presented with bleeding on probing, clinical attachment loss of 3 to $4 \mathrm{~mm}$, probing depth $\geq 5$ and $<7 \mathrm{~mm}$, and radiographic bone loss 16 to $30 \%$ were classified as generalized moderate chronic periodontitis. In addition, patients with greater than $30 \%$ of teeth presented with bleeding on probing, clinical attachment loss of $\geq 5 \mathrm{~mm}$, probing depth $\geq 7 \mathrm{~mm}$, and radiographic bone loss $>30 \%$ were classified as generalized severe chronic periodontitis. The control group were subjects without periodontitis. The inclusion criteria of subjects were between the ages of 30 to 65 , controlled or no systemic diseases, and non-smokers. The exclusion criteria were: 1) uncontrolled systemic diseases, such as uncontrolled diabetes (HbA1c $\geq 6.5 \%)$ and uncontrolled hypertension; 2$)$ recent history of myocardial infarction, cerebrovascular accident, transient ischemic attack, or coronary artery disease diagnosed in the past 6 months; 3) immunocompromised diseases; 4) BMI $<18.5$ or $\geq 40 \mathrm{~kg} / \mathrm{m}^{2}$; 5) pregnancy; 6) smokers; 7) receiving chemotherapy, radiotherapy, corticosteroid, anticoagulant, antiplatelet or nonsteroidal anti-inflammatory drugs; 8) antibiotic therapy within 6 months; 9) history of drug or substance abuse; or 10) patients received surgical or nonsurgical periodontal therapy for periodontitis within 2 years.

PRF collection: 
Intravenous access was obtained through the cubital region of the arm or dorsum of the hand for blood collection based on vessel size and accessibility. A total of $13 \mathrm{ml}$ of whole blood were collected from each subject. Three ml were sent to the Indiana University Health Pathology Lab for complete blood count (CBC) analysis. The remaining $10 \mathrm{ml}$ were centrifuged (Kendro Laboratory Products, Hanau, Germany) at $2700 \mathrm{rpm}$ (relative centrifuge force max was about $650 \mathrm{~g}$ ) for 12 minutes for PRF and serum collection. The collected sera were frozen at $-80^{\circ} \mathrm{C}$ for later proinflammatory cytokine (IL-1 $\beta$, IL-6, and TNF- $\alpha$ ) quantification by ELISA (R\&D Systems, MN, USA). After centrifugation, the PRF clots were harvested and compressed for 5 minutes in a PRF box (OSUNG MND Co, Kyonggi-do, Korea) into flattened PRF membranes. Membranes were trimmed into a uniform dimension of 15 x 20 $\mathrm{mm}^{2}$ from each PRF clot and weighed (Fisher Scientific Company, MA, USA) for standardization. The $15 \mathrm{x} 20 \mathrm{~mm}{ }^{2}$ membranes and the liquid exudates from the compressed PRF were frozen at $-80^{\circ} \mathrm{C}$ for later growth factor quantification. The samples were stored at $-80^{\circ} \mathrm{C}$ to minimize possible degradation of both the cells and the growth factor proteins of the PRF as much as possible. To avoid possible degradation of the proteins, the samples were stored no longer than 3 months before measurement.

\section{Growth factor quantification by ELISA:}

After collection of the samples, the $15 \times 20 \mathrm{~mm}^{2}$ membranes were incubated at $37^{\circ} \mathrm{C}$ in $5 \mathrm{ml}$ of saline, and at 1,24 , and 72 hours, eluted aliquots $(1.6 \mathrm{ml})$ were collected and frozen at $-80^{\circ} \mathrm{C}$ until analyzed. After 72 hours of incubation, the remaining membranes were digested to determine the amount of residual/unreleased growth factors from the membranes with $10 \mu \mathrm{g} / \mathrm{mL}$ trypsin (Worthington Biochemical Corporation, NJ, USA) for 24 hours. After trypsin digestion, $1 \mathrm{mg} / \mathrm{mL}$ soybean trypsin inhibitor (Worthington Biochemical Corporation, NJ, USA) was added to inhibit the trypsin. The PRF liquid exudates, the aliquots from PRF membrane incubation, and the digested membranes were analyzed by ELISA (R\&D Systems, MN, USA) according to the manufacturer's instructions for EGF, IGF-1, PDGF-BB, TGF- $\beta 1$, and VEGF detection and quantification.

Sample size calculation and statistical analysis:

Sample size calculation was done based on previous growth factors measurements from PRF by Kobayashi et al. [19], the standard deviations for the total growth factor release over 3 days are $235 \mathrm{pg} / \mathrm{mL}$ for TGF, $63 \mathrm{pg} / \mathrm{mL}$ for VEGF, $95 \mathrm{pg} / \mathrm{mL}$ for EGF, $33 \mathrm{pg} / \mathrm{mL}$ for IGF, and $155 \mathrm{pg} / \mathrm{mL}$ for PDGF-BB. With a sample size of 8 subjects per group, the study will have $80 \%$ power to detect TGF differences between groups of $354 \mathrm{pg} / \mathrm{mL}$ for TGF, $95 \mathrm{pg} / \mathrm{mL}$ 
for VEGF, $143 \mathrm{pg} / \mathrm{mL}$ for EGF, $50 \mathrm{pg} / \mathrm{mL}$ for IGF, and $234 \mathrm{pg} / \mathrm{mL}$ for PDGF-BB, assuming two-sided tests conducted at a $5 \%$ significance level.

Comparison of the effects of group and time on the growth factor measurements and the differences in growth factors present and released from membranes and exudates were made using repeated measures ANOVA. Comparisons between the test and control groups for differences in $\mathrm{CBC}$ and pro-inflammatory cytokine measurements were made using two-sample t-tests. Pearson correlation coefficients were calculated to assess the associations of the growth factor measurements with WBC counts, platelet counts, and pro-inflammatory cytokine concentrations. Correlation coefficients were compared between test and control groups using a z-test. A 5\% significance level was used for all tests.

\section{Results:}

\section{Patient characteristics:}

Ten subjects in the test group and nine in the control group were enrolled in the study (the demographic data of the groups are listed in Table 1). One subject from the test group and two from the control group were excluded from CBC analysis due to coagulation of the blood samples sent for analysis. Quantification of growth factors from the PRF were completed on all 19 subjects. The subjects were age matched and there was no statistically significant difference of mean age between groups (test $52.2 \pm 3.6$ years and control $52.8 \pm 9.2$ years, $\mathrm{p}=0.901$ ).

Growth factors from exudates and membranes between the groups:

Results of growth factor levels from exudates and membranes are listed in Figure 1 and Table 2. There were no significant differences for EGF ( $p=0.10)$, IGF-1 ( $p=0.83)$, PDGF-BB $(p=0.11)$, TGF- $\beta 1(p=0.83)$, and VEGF ( $p$ $=0.36$ ) measured from the exudates between the test and control groups. Also, there were no significant differences for EGF ( $p=0.19)$, IGF-1 $(p=0.09)$, PDGF-BB $(p=0.56)$, TGF- $\beta 1(p=0.81)$, and VEGF $(p=0.38)$ measured from the membranes between the test and control groups. Overall, there were no significant differences for EGF ( $p=$ $0.22)$ IGF-1 ( $p=0.42)$, PDGF-BB $(p=0.44)$, TGF- $\beta 1(p=0.85)$, and VEGF $(p=0.34)$ measured from the entire LPRF clots between the test and control groups. For EGF, PDGF-BB, TGF- $\beta 1$, and VEGF, the membranes contained significantly more growth factors than the exudates $(\mathrm{p}<0.01)$. However, for IGF-1 there were no significant differences measured from the exudates or the membranes of both the test and control groups $(p=0.52$ for the test group and $\mathrm{p}=0.33$ for the control group). 
Growth factors released over time between the groups:

$E G F$ :

Results of growth factors released over time are listed in Figure 2 and Table 3. Both test and control groups exhibited significantly lower EGF amounts released after 1 hour of incubation compared to 24 and 72 hours ( $\mathrm{p}<$ 0.05). Comparing the EGF being released from the membranes to that being entrapped within the remaining membranes (residual EGF), the majority of the EGF was released within 72 hours for both groups $(\mathrm{p}<0.05)$. The control group had significantly higher amounts of EGF release when measured after 1 hour of membrane incubation than the test group $(\mathrm{p}=0.00)$. In addition, the control group had significantly more residual EGF entrapped in the membranes than the test group $(\mathrm{p}=0.04)$.

$I G F-1:$

The control group had significantly higher amounts of IGF-1 release than the test group when measured after 1 hour of membrane incubation $(\mathrm{p}=0.04)$. Compared to 24 and 72 hours, the control exhibited the highest IGF-1 amount of release after 1 hour of incubation ( $\mathrm{p}<0.05$ ). For the test group, IGF-1 release at 24 hours was higher than 72 hours $(\mathrm{p}<0.05)$. For both the test and control groups, there were significantly greater amounts of released IGF-1 compared to membrane-retained IGF-1 $(\mathrm{p}<0.05)$.

$P D G F-B B$ :

Compared to 1 hour, both groups exhibited higher amount of PDGF-BB released after 24 and 72 hours of incubation $(\mathrm{p}<0.05)$. Comparing the PDGF-BB released from the membranes to that remaining entrapped within the remaining membranes, the majority of the growth factor was released for both groups $(\mathrm{p}<0.05)$. No significant differences were found comparing PDGF-BB measurements at 1, 24, 72 hours, and entrapped within the membranes between the test and the control groups $(\mathrm{p}>0.05)$.

TGF- $\beta 1$ :

The test group had a significantly higher amount of TGF- $\beta 1$ released when measured after 1 hour of membrane incubation $(\mathrm{p}=0.04)$. Compared to 1 hour, both groups exhibited higher amounts of TGF- $\beta 1$ released after 24 and 72 hours of incubation $(\mathrm{p}<0.05)$. Comparing the amount of TGF- $\beta 1$ released from the membranes to that entrapped within the remaining membranes, the majority of the growth factor was released for both groups within 72 hours ( $p$ $<0.05$ ). No significant differences were found comparing TGF- $\beta 1$ measurements at $1,24,72$ hours, and entrapped within the membranes between the test and the control groups $(\mathrm{p}>0.05)$. 
$V E G F$ :

For both the test and the control groups, the VEGF amount released at 24 and 72 hours was significantly higher than 1 hour $(\mathrm{p}<0.05)$. Comparing the amount of VEGF released from the membranes to that entrapped within the remaining membranes, the majority of the growth factor was released for both groups $(\mathrm{p}<0.05)$. No significant differences were found comparing VEGF measurements at 1, 24, 72 hours, and entrapped within the membranes between the test and the control groups $(\mathrm{p}>0.05)$.

Comparison of CBC and pro-inflammatory cytokines between the groups:

The test group had significantly higher WBC counts than the control group $(\mathrm{p}=0.04)$, but no other statistically significant group differences were found among the $\mathrm{CBC}$ results, including the platelet count $(\mathrm{p}=0.33)$. However, there were no significant correlations of the growth factors studied with the WBC counts for both test and control subjects ( $p>0.05$ ). In addition, no statistically significant group differences were found when comparing the amount of IL-1 $\beta(p=0.93)$, IL-6 ( $p=0.62)$, and TNF- $\alpha(p=0.29)$ between the test and the control groups (Table 4).

\section{Discussion:}

The results from the current study revealed that the amounts of growth factors (EGF, IGF-1, PDGF-BB, TGF- $\beta 1$, and VEGF) present in the liquid exudates and released from the PRF membranes had wide range of values between individuals, but there were no significant differences between the test and the control groups. This suggests that the amounts of growth factors studied were not affected by the periodontal disease status of the subjects.

Also, except for IGF-1, there were much higher concentrations of EGF, PDGF-BB, TGF- $\beta 1$, and VEGF in the PRF membranes compared to the exudates. Such findings support the clinical use of PRF membranes as an autologous growth factor delivery vehicle. However, since growth factors were still detected in the PRF exudates, and the IGF-1 amounts from the exudates was comparable to that from the membranes, hydrating graft materials with PRF exudates in combination with utilizing the PRF membranes can maximize the benefits from the entire PRF clot. Furthermore, relative to the release of growth factors from the PRF membranes, IGF-1 tended to be released almost immediately, while the amounts of EGF, PDGF-BB, TGF- $\beta 1$, and VEGF were mostly released within the first 24 hours that reached plateaus. Very little additional growth factor release by 72 hours was observed that could be due to either the plateau was reached or the nature of our study design that the PRF membranes were digested by trypsin at the end of the study. There were occasional instances when the total growth factor released at later time periods 
was less than previous time periods (Table 3). This may have been due to the fact that the majority of the growth factors were released and no more were available. Additionally, there were still small amounts of growth factors that remained entrapped in the remaining membranes at 72 hours. Although it was found that the majority of the growth factors were released within the first 24 hours, the entrapped growth factors still detected after 72 hours of incubation may support the clinical application of PRF membranes where some growth factors may be still released over an extended period at the surgical site until eventual fibrinolysis of the membranes. The present study used saline to incubate the samples. Although culture media can ensure viability of cells, the growth factors measured are released from platelets rather than the trapped leukocytes [16]. Cell viability would not be expected to have an effect on the amounts of measured growth factors, but further study is warranted to verify this. The results revealed that the control group had significantly higher amounts of EGF release when measured at 1 hour after membrane incubation $(p=0.00)$ and significantly more residual EGF entrapped in the membranes than the test group $(p=0.04)$.

Furthermore, there were significantly greater amounts of IGF-1 released at 1 hour by the control group, compared to the test group $(\mathrm{p}=0.04)$. Since EGF and IGF-1 regulates cell differentiation and proliferation in wound healing [16], the wound healing capacity from patients with active inflammation from chronic periodontitis may be downregulated. However, the results demonstrated that the test group had significantly higher amounts of TGF- $\beta 1$ release at 1 hour from the PRF membranes $(\mathrm{p}=0.04)$. The initial elevation of TGF- $\beta 1$ may be partially explained, as TGF$\beta 1$ promotes inflammatory cell recruitment and inflammation increases TGF- $\beta 1$ response [16, 20$].$

Although the results exhibited higher WBC levels in the test group, there were no correlations found between the WBC numbers and the amounts of the growth factors between the groups. Possible explanation of such observation could be that with the present PRF preparation protocol, WBC are less entrapped within the PRF compared to other centrifuge protocols which tubes of blood are centrifuged at a slower speed [21]. Furthermore, as the subjects in the test group were patients with untreated generalized moderate to severe chronic periodontitis, the inflammatory status represented by elevated leukocytes was expected [22]. While no other differences were found from the results of the CBC analysis between groups, this might be expected as all subjects were systemically healthy or had controlled systemic diseases. Even though a previous study by Gorska et al. revealed elevation of pro-inflammatory cytokines with periodontitis, they found higher cytokine concentrations in gingival crevicular fluid compared to the systemic circulation [2]. In the present study, the amount of pro-inflammatory cytokines of the subjects at a systemic level were determined by the collected serum from the centrifuged blood. As chronic periodontitis primarily causes 
inflammation of the periodontium, it is understandable that systemic inflammatory responses may not reflect those found at the local level.

There are several PRF preparation protocols. The PRF centrifugation protocol used in the present study has been reported to entrap leukocytes and platelets within the fibrin clot [23]. However, the source of EGF, IGF-1, PDGFBB, TGF- $\beta 1$, and VEGF are primarily from the platelets rather than the leukocytes [16]. Therefore, it is not surprising to find that there were no correlations between the WBC and the amounts of growth factors from PRF. No previous research could be found that evaluated the influence of $\mathrm{CBC}$ or chronic periodontitis status on the quantity or release of growth factors from PRF. Kobayashi et al. evaluated the comparative release of growth factors from PRF, advanced-PRF, and platelet-rich plasma from healthy individuals. They quantified the amounts and release of growth factors including PDGF-AA, PDGF-AB, PDGF-BB, TGF- $\beta$, VEGF, EGF, and IGF released from platelet concentrates by ELISA for up to 10 days. The amounts of growth factors quantified are similar to the results of the present study, while large standard deviations were found for both studies. The authors suggested that several patient-related factors might affect growth factor release, so that there may be a wide variability of the amounts of growth factors released. Although the results of the current study were similar to that of the Kobayashi study, direct comparisons should be avoided as the study methods were not the same. Kobayashi et al. evaluated growth factors from PRF clots, while the present study used compressed PRF membranes as PRF is commonly used in the membranous form $[24,25]$. The current study also digested the remaining membranes to quantify the amount of growth factors not released and remaining entrapped within the fibrin clots.

The strengths of the current study design were that after separation of the liquid exudates from the PRF membranes, comparisons of growth factors from membranes and exudates were possible. Also, digestion of the remaining membranes with trypsin after incubation to quantify the growth factors entrapped in the remaining fibrin clot which may reflect contained growth factor release during the clinical degradation of the PRF membranes.

In conclusion, the amounts of growth factors released from PRF show variation between patients. However, the amounts of growth factors from the PRF exudates or the PRF membranes had no statistically significant differences between the test and the control groups. Except IGF-1, most of the growth factors were from the membranes rather than the exudates. The majority of the growth factors were released within 24 hours of incubation and the small remaining amounts of growth factors continued to be released up to 72 hours. The low levels of residual growth factors noted after 72 hours suggests a continued release until eventual fibrinolysis of the PRF membranes occurs. 
$\mathrm{CBC}$ results indicated that the test group had significantly higher WBC $(\mathrm{p}<0.05)$. However, there was no significant correlation between the WBC counts and the amounts of any of the tested growth factors from PRF. Within the limitations of the current study, one can conclude that PRF can be utilized as an autologous source of growth factors not affected by periodontal condition and the numbers of WBC of the healthy patients. Large standard errors found from this study suggests further studies with larger subjects is indicated. 


\section{Compliance with Ethical Standards}

\section{Conflict of Interest:}

Dr. Jennifer Chang declares that she has no conflict of interest. Dr. Steven B. Blanchard declares that he has no conflict of interest. Dr. L. Jack Windsor declares that he has no conflict of interest. Dr. Richard L. Gregory declares that he has no conflict of interest. Dr. Yusuke Hamada declares that he has no conflict of interest.

\section{Funding:}

The work was supported by a Young Researcher Grant provided by the Osteology Foundation, Switzerland (project number: 16-142), and the Indiana University School of Dentistry Graduate Student Research Committee.

\section{Ethical approval:}

All procedures performed in studies involving human participants were in accordance with the ethical standards of the institutional research committee.

\section{Informed consent:}

Informed consent was obtained from all individual participants included in the study. 


\section{References:}

1. Eke, P., Dye, B., Wei, L., Thornton-Evans, G. \& Genco, R. (2012) Prevalence of periodontitis in adults in the United States: 2009 and 2010. Journal of Dental Research 91, 914-920.

2. Górska, R., Gregorek, H., Kowalski, J., Laskus-Perendyk, A., Syczewska, M. \& Madaliński, K. (2003) Relationship between clinical parameters and cytokine profiles in inflamed gingival tissue and serum samples from patients with chronic periodontitis. Journal of Clinical Periodontology 30, 1046-1052.

3. Avila-Ortiz, G., De Buitrago, J.G. \& Reddy, M.S. (2015) Periodontal regeneration-Furcation defects: A systematic review from the AAP regeneration workshop. Journal of Periodontology 86(suppl.), S108-S130.

4. Kao, R.T., Nares, S. \& Reynolds, M.A. (2015) Periodontal regeneration-intrabony defects: a systematic review from the AAP regeneration workshop. Journal of Periodontology 86 (suppl.), S77-S104.

5. Choukroun, J., Diss, A., Simonpieri, A., Girard, M., Schoeffler, C., Dohan, S.L., Dohan, A.J.J., Mouhyi, J. \& Dohan, D. (2006) Platelet-rich fibrin (PRF): a second-generation platelet concentrate. Part IV: clinical effects on tissue healing. Oral Surgery Oral Medicine Oral Pathology Oral Radiology and Endodonthics 101, e56-60.

6. Choukroun, J., Diss, A., Simonpieri, A., Girard, M., Schoeffler, C., Dohan, S.L., Dohan, A.J.J., Mouhyi, J. \& Dohan, D. (2006) Platelet-rich fibrin (PRF): a second-generation platelet concentrate. Part V: histologic evaluations of PRF effects on bone allograft maturation in sinus lift. Oral Surgery Oral Medicine Oral Pathology Oral Radiology and Endodonthics 101, 299-303.

7. Dohan, D.M., Choukroun, J., Diss, A., Dohan, S.L., Dohan, A.J.J., Mouhyi, J. \& Gogly, B. (2006) Platelet-rich fibrin (PRF): a second-generation platelet concentrate. Part I: technological concepts and evolution. Oral Surgery Oral Medicine Oral Pathology Oral Radiology and Endodonthics 101, e37-44.

8. Dohan, D.M., Choukroun, J., Diss, A., Dohan, S.L., Dohan, A.J.J., Mouhyi, J. \& Gogly, B. (2006) Platelet-rich fibrin (PRF): a second-generation platelet concentrate. Part II: platelet-related biologic features. Oral Surgery Oral Medicine Oral Pathology Oral Radiology and Endodonthics 101, e45-50.

9. Dohan, D.M., Choukroun, J., Diss, A., Dohan, S.L., Dohan, A.J.J., Mouhyi, J. \& Gogly, B. (2006) Platelet-rich fibrin (PRF): a second-generation platelet concentrate. Part III: leucocyte activation: a new feature for platelet concentrates? Oral Surgery Oral Medicine Oral Pathology Oral Radiology and Endodonthics 101, e51-55. 
10. Kobayashi, E., Fluckiger, L., Fujioka-Kobayashi, M., Sawada, K., Sculean, A., Schaller, B. \& Miron, R.J. (2016) Comparative release of growth factors from PRP, PRF, and advanced-PRF. Clinical Oral Investigation 20, 2353-2360.

11. Masuki, H., Okudera, T., Watanebe, T., Suzuki, M., Nishiyama, K., Okudera, H., Nakata, K., Uematsu, K., Su, C. \& Kawase, T. (2016) Growth factor and pro-inflammatory cytokine contents in platelet-rich plasma (PRP), plasma rich in growth factors (PRGF), advanced platelet-rich fibrin (A-PRF), and concentrated growth factors (CGF). International Journal of Implant Dentistry $2,19$.

12. Singh, A., Kohli, M. \& Gupta, N. (2012) Platelet rich fibrin: a novel approach for osseous regeneration. Journal of Maxillofacial and Oral Surgery 11, 430-434.

13. Sharma, A. \& Pradeep, A.R. (2011) Autologous platelet-rich fibrin in the treatment of mandibular degree II furcation defects: a randomized clinical trial. Journal of Periodontology 82, 1396-1403.

14. Thorat, M., Pradeep, A.R. \& Pallavi, B. (2011) Clinical effect of autologous platelet-rich fibrin in the treatment of intra-bony defects: a controlled clinical trial. Journal of Clinical Periodontology 38, 925-932.

15. Miron, R.J., Dham, A., Dham, U., Pikos, M.A. \& Sculean, A. (2018) The effect of age, gender, and time between blood draw and start of centrifugation on the size outcomes of platelet-rich fibrin (PRF) membranes. Clinical oral investigations 2, 1-7.

16. Larjava, H. (2012) Oral wound healing: cell biology and clinical management. 1st edition, p. 261-286. John Wiley \& Sons.

17. Sabharwal, A., Gomes-Filho, I. S., Stellrecht, E. \& Scannapieco, F. A. (2018) Role of periodontal therapy in management of common complex systemic diseases and conditions: An update. Periodontology 2000 78, 212226.

18. PERIODONTITIS, O. (2015) American Academy of Periodontology task force report on the update to the 1999 classification of periodontal diseases and conditions. Journal of Periodontology 86, 835-838.

19. Smith, P.C., Martínez, C., Cáceres, M. \& Martínez, J. (2015) Research on growth factors in periodontology. Periodontology 2000 67, 234-250.

20. Choukroun, J., \& Ghanaati, S. (2018) Reduction of relative centrifugation force within injectable platelet-richfibrin (PRF) concentrates advances patients' own inflammatory cells, platelets and growth factors: the first 
introduction to the low speed centrifugation concept. European Journal of Trauma and Emergency Surgery 44, $87-95$.

21. Flemmig, T.F. (1999) Periodontitis. Annals of Periodontology 4, 32-37.

22. Ehrenfest, D.M.D., Rasmusson, L. \& Albrektsson, T. (2009) Classification of platelet concentrates: from pure platelet-rich plasma (P-PRP) to leucocyte-and platelet-rich fibrin (L-PRF). Trends in biotechnology 27, 158167.

23. Del Corso, M., Vervelle, A., Simonpieri, A., Jimbo, R., Inchingolo, F., Sammartino, G. \& Ehrenfest D.M.D. (2012) Current knowledge and perspectives for the use of platelet-rich plasma (PRP) and platelet-rich fibrin (PRF) in oral and maxillofacial surgery part 1: Periodontal and dentoalveolar surgery. Current Pharmaceutical Biotechnology 13, 1207-1230.

24. Simonpieri, A., Del Corso, M., Vervelle, A., Jimbo, R., Inchingolo, F., Sammartino, G. \& Ehrenfest D.M.D. (2012) Current knowledge and perspectives for the use of platelet-rich plasma (PRP) and platelet-rich fibrin (PRF) in oral and maxillofacial surgery part 2: Bone graft, implant and reconstructive surgery. Current Pharmaceutical Biotechnology 13, 1231-1256. 
Table 1. Demographic data of the subjects

\begin{tabular}{|c|c|c|c|c|}
\hline Groups & $\begin{array}{l}\text { Mean age } \\
\text { (year } \pm \text { SD) }\end{array}$ & Patient \# & Age & Gender \\
\hline \multirow[t]{9}{*}{ Test $(n=9)$} & $52.2 \pm 10.8$ & 1 & 65 & Male \\
\hline & & 2 & 60 & Male \\
\hline & & 3 & 41 & Female \\
\hline & & 4 & 34 & Male \\
\hline & & 5 & 61 & Male \\
\hline & & 6 & 54 & Male \\
\hline & & 7 & 48 & Male \\
\hline & & 8 & 62 & Female \\
\hline & & 9 & 63 & Female \\
\hline \multirow{10}{*}{$\begin{array}{l}\text { Control } \\
(n=10)\end{array}$} & $52.8 \pm 9.2$ & A & 60 & Female \\
\hline & & $\mathrm{B}$ & 64 & Female \\
\hline & & $\mathrm{C}$ & 51 & Female \\
\hline & & $\mathrm{D}$ & 37 & Male \\
\hline & & $\mathrm{E}$ & 56 & Female \\
\hline & & $\mathrm{F}$ & 54 & Female \\
\hline & & G & 55 & Male \\
\hline & & $\mathrm{H}$ & 62 & Male \\
\hline & & I & 39 & Male \\
\hline & & $\mathrm{J}$ & 51 & Male \\
\hline
\end{tabular}


Table 2. Growth factor measurements from PRF liquid exudates compressed from PRF, PRF membranes, and the combination of both between the groups at the end of the study

\section{Median (Q1-Q3)}

(pg/PRF)

\begin{tabular}{|c|c|c|c|}
\hline Growth factor & Control group & Test group & $P$ value \\
\hline EGF (E) & $42.39(33.67-117.87)$ & $94.96(55.09-128.29)$ & 0.10 \\
\hline EGF (M) & $2437.15(2155.65-2762.52)^{*}$ & $1846.43(1704.14-1976.76)^{*}$ & 0.19 \\
\hline IGF-1 (E) & 76901.57 (54503.36-103687.75) & $89387.58(55075.50-102111.30)$ & 0.83 \\
\hline IGF-1 (M) & $78782.92(69528.38-97154.04)$ & $63111.30(49633.97-73039.87)$ & 0.09 \\
\hline PDGF-BB (E) & $492.31(227.59-731.78)$ & $959.82(779.09-1035.78)$ & 0.11 \\
\hline PDGF-BB (M) & $3457.91(1919.87-4503.78)^{*}$ & $3792.63(3254.03-4887.49)^{*}$ & 0.56 \\
\hline TGF- $\beta 1$ (E) & $953.12(536.52-1449.39)$ & $1034.42(913.33-1334.61)$ & 0.83 \\
\hline TGF- $\beta 1$ (M) & $7859.79(7252.62-9463.45)^{*}$ & $6896.36(6076.73-7962.62)^{*}$ & 0.81 \\
\hline VEGF (E) & $169.11(103.29-214.56)$ & $95.80(54.61-132.00)$ & 0.36 \\
\hline VEGF (M) & $1008.92(699.31-1158.00)^{*}$ & $532.96(433.19-1196.11)^{*}$ & 0.38 \\
\hline
\end{tabular}

Q1: 25 percentile, Q3: 75 percentile, (E): PRF exudates, (M): PRF membranes.

${ }^{*}$ Growth factors from the membranes were significantly higher than the exudates for EGF, PDGF-BB, TGF- $\beta 1$, and VEGF ( $\mathrm{p}<0.01$, calculated based on means and standard errors between groups). 
Table 3. Growth factors released over time between the groups

Median (Q1-Q3)

(pg/PRF)

$\begin{array}{llll}\text { Growth factor } & \text { Control group } & \text { Test group } & \text { P value }\end{array}$

(Elution time-hours)

\begin{tabular}{|c|c|c|c|}
\hline EGF (1) & $828.87(636.64-1101.70)^{* *}$ & $149.87(15.39-410.66)^{* *}$ & $0.00^{*}$ \\
\hline EGF (24) & $1691.04(1182.46-2373.72)$ & $1519.14(1262.39-1819.70)$ & 0.89 \\
\hline EGF (72) & $1703.15(1135.13-2150.89)$ & $1428.66(1386.88-1693.82)$ & 0.61 \\
\hline EGF (Remaining) & $486.43(404.85-737.94)^{\#}$ & $310.03(275.48-406.64)^{\#}$ & $0.04^{*}$ \\
\hline IGF-1 (1) & $6782.95(45442.62-71561.27)$ & $38527.51(29429.39-42807.63)$ & $0.04^{*}$ \\
\hline IGF-1 (24) & $47102.49(41699.75-63322.27)^{* *}$ & $41727.26(30805.39-46692.09)$ & 0.11 \\
\hline IGF-1 (72) & $50405.00(38485.60-54728.60)^{* *}$ & $36757.76(32300.42-45706.78)^{* *}$ & 0.06 \\
\hline IGF-1 (Remaining) & $35492.03(23239.62-41824.78)^{\#}$ & $23083.58(18096.36-37868.89)^{\#}$ & 0.36 \\
\hline PDGF-BB (1) & $323.64(240.00-522.96)^{* *}$ & $731.76(270.66-1647.11)^{* *}$ & 0.11 \\
\hline PDGF-BB (24) & $2558.51(1604.59-4313.83)$ & $2706.48(2475.05-3688.59)$ & 0.69 \\
\hline PDGF-BB (72) & $3119.96(1716.31-4327.78)$ & $3680.12(3172.33-4805.51)$ & 0.50 \\
\hline PDGF-BB (Remaining) & $282.98(187.94-415.23)^{\#}$ & $288.63(112.50-408.99)^{\#}$ & 0.53 \\
\hline TGF-ß1 (1) & $893.04(581.26-1093.58)^{* *}$ & $1684.36(651.15-2644.86)^{* *}$ & $0.04^{1}$ \\
\hline TGF- $\beta 1$ (24) & $6477.30(5264.97-7594.26)$ & $5309.62(4356.53-5446.23)$ & 0.75 \\
\hline TGF- $\beta 1$ (72) & 6936.05 (6289.64-7167.02) & 5963.16 (4822.08-6566.78) & 0.64 \\
\hline TGF-p1 (Remaining) & $1201.81(459.26-2443.42)^{\#}$ & $1104.49(627.08-2167.80)^{\#}$ & 0.86 \\
\hline VEGF (1) & $123.14(82.37-217.75)^{* *}$ & $95.38(79.37-200.00)^{* *}$ & 0.69 \\
\hline
\end{tabular}




$\begin{array}{cccc}\text { VEGF (24) } & 712.16(431.14-823.81) & 346.31(315.22-723.64) & 0.36 \\ \text { VEGF (72) } & 700.17(458.40-753.43) & 326.83(298.62-756.75) & 0.44 \\ \text { VEGF (Remaining) } & 288.50(136.27-294.85)^{\#} & 253.91(134.56-279.60)^{\#} & 0.49\end{array}$

Q1: 25 percentile, Q3: 75 percentile, (1): Growth factors released detected at 1 hour, (24): growth factors released detected at 24 hours, (72): Growth factors released detected at 72 hours, (Remaining): growth factors remaining entrapped within the PRF membranes.

${ }^{*} \mathrm{P}$ value $<0.05$ : Significant differences between the test and control groups. (P value calculated based on means and standard errors between groups.)

${ }^{* *}$ Significantly lower within the test and control groups $(\mathrm{p}<0.05)$.

\# Significantly lower growth factors entrapped within the membranes $(p<0.05)$. 
Table 4. CBC and pro-inflammatory cytokines between the groups

\section{Median (Q1-Q3)}

\begin{tabular}{cccc} 
& Control group & Test group & P value \\
\hline WBC $\left(\mathbf{c e l l s} / \mathbf{m m}^{\mathbf{3}}\right)$ & $5.60(5.25-6.55)$ & $7.80(6.38-8.78)$ & $0.04^{*}$ \\
Platelet $\left(\mathbf{c e l l s} / \mathbf{m m}^{3}\right)$ & $232.00(216.00-257.75)$ & $242.50(160.75-288.00)$ & 0.38 \\
IL-1 $\boldsymbol{\beta}(\mathbf{p g} / \mathbf{m L})$ & $3.88(2.69-5.25)$ & $4.42(2.94-4.78)$ & 0.93 \\
IL-6 $(\mathbf{p g} / \mathbf{m L})$ & $1.48(0.82-2.98)$ & $3.99(3.78-10.06)$ & 0.62 \\
TNF- $\alpha(\mathbf{p g} / \mathbf{m L})$ & $7.98(4.16-14.62)$ & $11.82(11.18-14.92)$ & 0.29
\end{tabular}

Q1: 25 percentile, Q3: 75 percentile.

${ }^{*}$ The test group had significantly higher WBC than the control group $(\mathrm{p}<0.05$, calculated based on means and standard errors between groups). 
Figure 1. Growth factor measurements from PRF liquid exudates, PRF membranes, and the combination of both between the groups at the end of the study

${ }^{*}$ Growth factors from the PRF membranes were significantly higher than the exudates $(\mathrm{p}<0.01)$.

\section{Figure 2. Growth factors released from PRF membranes over time between the groups}

${ }^{*}$ Both test and control groups exhibited significantly lower EGF amounts released after 1 hour of incubation compared to 24 and 72 hours $(\mathrm{p}<0.05)$.

${ }^{+}$The majority of the EGF was released within 72 hours for both groups $(\mathrm{p}<0.05)$.

! The control group had significantly higher amounts of EGF release when measured after 1 hour of membrane incubation and more residual EGF entrapped in the membranes than the test group $(\mathrm{p}<0.05)$.

(a) The control group had significantly higher amounts of IGF-1 release than the test group when measured after 1 hour of membrane incubation and exhibited the highest IGF-1 amount of release after 1 hour of incubation ( $\mathrm{p}<$ $0.05)$.

\# For the test group, IGF-1 release at 24 hours was higher than 72 hours $(\mathrm{p}<0.05)$.

${ }^{\$}$ For both the test and control groups, there were significantly greater amounts of released IGF-1 compared to membrane-retained IGF-1 $(\mathrm{p}<0.05)$. 
${ }^{\%}$ Compared to 1 hour, both groups exhibited higher amount of PDGF-BB, TGF- $\beta 1$, and VEGF released after 24 and 72 hours of incubation $(\mathrm{p}<0.05)$.

${ }^{\&}$ Comparing the released from the membranes to that remaining entrapped within the remaining membranes, the majority of PDGF-BB, TGF- $\beta 1$, and VEGF were released for both groups $(\mathrm{p}<0.05)$.

\section{Figure 3. Comparison of $\mathrm{CBC}$ and pro-inflammatory cytokines between the groups}

${ }^{*}$ The test group had significantly higher WBC than the control group $(\mathrm{p}<0.05)$. 